\title{
The absorption of aspirin is reduced after coronary artery bypass grafting
}

\author{
Andreas Lund Hattesen, MS, ${ }^{a}$ Ivy Susanne Modrau, MD, DrMedSc, ${ }^{b}$ Dorthe Viemose Nielsen, MD, PhD, \\ and Anne-Mette Hvas, MD, $\mathrm{PhD}^{\mathrm{a}, \mathrm{d}, \mathrm{e}}$
}

\begin{abstract}
Background: Despite treatment with the antiplatelet drug aspirin, some patients who undergo coronary artery bypass grafting (CABG) experience a thromboembolic event in the early postoperative period. The etiology of a reduced aspirin effect after CABG is still unsolved. Hence, we investigated whether patients who undergo $\mathrm{CABG}$ have a reduced absorption of aspirin after surgery, measured as levels of plasma salicylic acid (SA).
\end{abstract}

Methods: In total, 53 patients were included between October 2015 and May 2018. Blood samples where obtained at the day before surgery, and at the first (day 1) and second (day 2) postoperative day. A subgroup of 10 patients had additional blood samples obtained on day 1. Plasma SA was measured using liquid chromatography-electrospray-ionization tandem-mass spectrometry. Multiplate Analyzer (Roche, Roche Diagnostics, Mannheim, Germany) and VerifyNow Aspirin Test (Accumetrics, San Diego, Calif) were used to assess platelet aggregation as a measurement of the antiplatelet effect of aspirin.

Results: A comparison of the difference in change of SA concentration between the day before surgery with day 1 and day 2 showed a significantly reduced increase in SA plasma levels after intake of aspirin at day 1 and day 2 after surgery $(P<.0001)$, including a significantly reduced SA increase in the subgroup at 2,3 , and 4 hours after intake of aspirin. Corresponding with a reduced effect of aspirin, neither Multiplate Analyzer arachidonic acid, nor VerifyNow Aspirin Test showed a significant reduction in platelet aggregation 1 hour after intake of aspirin at day $1(P<.0001)$. This also accounted for the subgroup 2,3 , and 4 hours after aspirin intake on day 1 . At day 2 the same pattern as preoperatively was detected.

Conclusions: After CABG, the SA level and the effect of aspirin were reduced in the immediate postoperative period. (J Thorac Cardiovasc Surg 2019;157:1059-68)

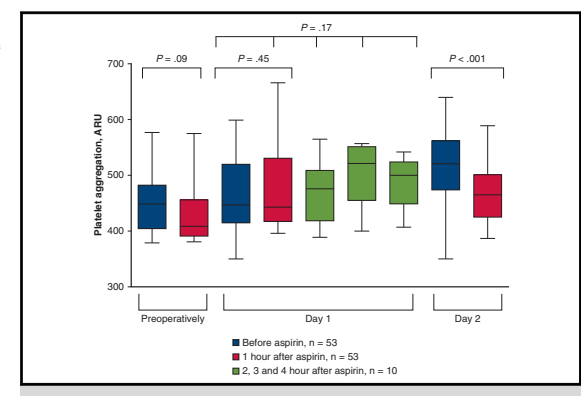

Potential explanation to the early postoperative thromboembolic events in CABG patients.

\section{Central Message}

Absorption of low-dose aspirin is significantly reduced in the immediate postoperative period of $\mathrm{CABG}$ with a corresponding reduced antiplatelet effect.

Perspective

A reduced absorption of low-dose aspirin and a corresponding reduced antiplatelet effect can theoretically be the explanation for the incidence of thromboembolic events in the early postoperative period of CABG. Future studies are needed to clarify a possible clinical superiority of intravenous treatment with aspirin compared with oral treatment.

See Commentary on page 1069.

\footnotetext{
From the a Department of Biochemistry, Centre for Haemophilia and Thrombosis, ${ }^{\mathrm{b}}$ Department of Cardiothoracic and Vascular Surgery, ${ }^{\mathrm{c}}$ Department of Anaesthesiology and Intensive Care, ${ }^{\mathrm{d}}$ Department of Clinical Biochemistry, and ${ }^{\mathrm{e}}$ Department of Clinical Medicine, Aarhus University Hospital, Aarhus, Denmark.

The study was funded by Novo Nordisk, Eva og Henry Frænkels Mindefond, Murermester Lauritz Peter Christensen og hustru Kirsten Sigrid Christensens Fond and Friedrich Wilhelm Frank og hustru Angelina Franks Mindelegat.

Received for publication Oct 19, 2017; revisions received July 14, 2018; accepted for publication Aug 7, 2018; available ahead of print Nov 3, 2018.

Address for reprints: Anne-Mette Hvas, MD, PhD, Department of Clinical Biochemistry, Aarhus University Hospital Palle Juul-Jensens Boulevard 99, DK-8200 Aarhus N, Denmark (E-mail: am.hvas@dadlnet.dk). $0022-5223 / \$ 36.00$

Copyright (C) 2018 Published by Elsevier Inc. on behalf of The American Association for Thoracic Surgery

https://doi.org/10.1016/j.jtcvs.2018.08.088
}

For decades, the antiplatelet drug acetylsalicylic acid (ASA, aspirin) has been a front-line medication for primary and secondary prophylaxis in coronary artery disease. The current guideline for myocardial revascularization recommends pre- and postoperative treatment with low-dose

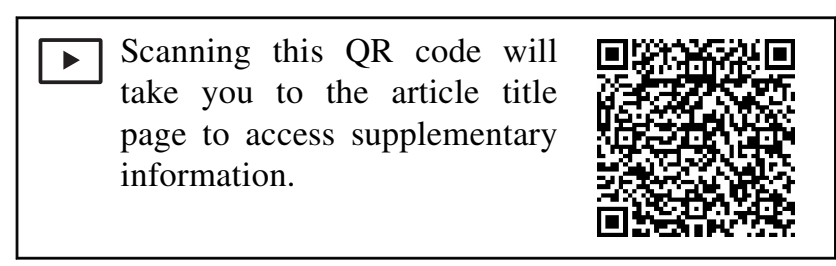




$\begin{array}{ll}\text { Abbreviations and } & \text { Acronyms } \\ \text { ASA } & =\text { acetylsalicylic acid } \\ \text { ASPI } & =\text { arachidonic acid } \\ \text { CABG } & =\text { coronary artery bypass grafting } \\ \text { COL } & =\text { collagen } \\ \text { COX } & =\text { cyclooxygenase } \\ \text { CPB } & =\text { cardiopulmonary bypass } \\ \text { LC-ESI-MS/MS } & =\text { liquid chromatography- } \\ & \text { electrospray-ionization-tandem } \\ & \text { mass spectrometry } \\ \text { SA } & =\text { salicylic acid } \\ \text { Tx } & =\text { thromboxane }\end{array}$

aspirin to prevent thromboembolic events in patients who undergo coronary artery bypass grafting $(\mathrm{CABG}){ }^{1}$

After ingestion, ASA quickly transforms into the inactive metabolite salicylic acid (SA). ASA has a plasma half-life of approximately 20 minutes, whereas SA has a half-life of approximately 3 hours and is stable in plasma. ${ }^{2}$ The antiplatelet effect of aspirin occurs by irreversible inhibition of platelet cyclooxygenase (COX)- 1 and COX-2. The enzyme inhibition blocks the formation of thromboxane (Tx) $\mathrm{A}_{2}$, and thereby inhibits the ability of the platelets to aggregate. ${ }^{3}$ Previously, measurement of the efficacy of aspirin has been conducted by either measuring the more stable derivate of $\mathrm{TxA}_{2}, \mathrm{TxB}_{2}$, or by various platelet aggregation tests. ${ }^{4}$

Despite aspirin prophylaxis, the risk of major adverse events is increased in the early postoperative period after CABG; nonfatal myocardial infarction occurs in $>2 \%$ of these patients in-hospital, ${ }^{5}$ and the incidence of perioperative stroke is approximately $1.5 \% .{ }^{6}$ Notably, a study conducted by Likosky and colleagues showed that most strokes happen the first or second day after surgery. ${ }^{7}$

The relatively high incidence of thromboembolic events in the early postoperative course after CABG remains inexplicable. However, several studies have shown a reduced effect of aspirin measured according to reduced platelet aggregation in the early postoperative period after coronary bypass surgery. ${ }^{8}$ A meta-analysis concluded that the reduced antiplatelet effect of aspirin after CABG is associated with a significant increased risk of subsequent thromboembolic events compared with patients with optimal aspirin effect. ${ }^{9}$

There is no consensus regarding the prevalence, duration, clinical effect, or etiology of a reduced aspirin effect after CABG. To the best of our knowledge, no previous study has investigated whether the absorption of aspirin is impaired after CABG.

Thus, the aim of the present study was to investigate the absorption of aspirin and the platelet effect of aspirin in the early postoperative period after CABG. Our primary hypothesis was that the absorption of aspirin, reflected by the level of SA, is reduced in the early postoperative period after $\mathrm{CABG}$ compared with absorption before surgery.

\section{METHODS \\ Study Population}

A total of 53 patients were included between October 2015 and May 2018 at the Department of Cardiothoracic and Vascular Surgery, Aarhus University Hospital, Denmark. Inclusion criteria were: elective isolated CABG, age 18 years or older, and daily prophylactic treatment with low-dose nonenteric-coated aspirin ( $75 \mathrm{mg}$ daily). Patients were excluded from the study if they met any of the following criteria at admission: contraindication to pause aspirin treatment on the day of surgery, a platelet count $<100 \times 10^{9} / \mathrm{L}$ or $>450 \times 10^{9} / \mathrm{L}$, acute coronary syndrome with indication of revascularization within 24 hours, concurrent cardiac surgery other than revascularization, intake of ticagrelor or clopidogrel within 5 days before surgery, and intake of non-vitamin $\mathrm{K}$ oral anticoagulants or vitamin $\mathrm{K}$ antagonists within 2 days before surgery. Patients who received transfusion of platelet concentrate intra- or postoperatively were excluded and replaced. All clinical information was obtained from systematic interviews and medical records.

\section{Study Design}

The present study was a prospective observational study on patients who underwent elective isolated CABG. Patients were scored preoperatively according to the European System for Cardiac Operative Risk Evaluation Score, a percentage score to predict mortality after cardiac surgery. ${ }^{10}$

All patients received prophylactic treatment with nonenteric-coated aspirin $75 \mathrm{mg}$ daily before surgery. This treatment was discontinued on the day of surgery and resumed on the first postoperative day. Blood samples were collected before and 1 hour after the intake of aspirin on the day before surgery (preoperatively), and on the first (day 1) and second (day 2) postoperative day (Figure 1). A substudy was conducted implying that 10 patients had blood samples obtained before and 1,2,3, and 4 hours after the intake of aspirin on day 1 . Aspirin was administered orally and the intake was observed each time.

All patients underwent conventional CABG using cardiopulmonary bypass (CPB). All patients received standard anesthesia comprising a total of sufentanil $4-5 \mu \mathrm{g} / \mathrm{kg}$ intraoperatively along with a bolus of rocuronium $0.6 \mathrm{mg} / \mathrm{kg}$. Anesthesia was maintained with propofol infusion $100-400 \mathrm{mg} / \mathrm{h}$. No patients received epidural anesthesia. According to current guidelines, all patients received a prophylactic standard dose of tranexamic acid intraoperatively. Unfractionated heparin at a dose of $300 \mathrm{IU} / \mathrm{kg}$ was administered after harvest of the mammary artery and additional boluses were given as required to maintain an activated clotting time of $>400$ seconds. Protamine was administered at a dose of $1 \mathrm{mg}$ per given $100 \mathrm{IU}$ of heparin at the end of CPB to reverse the heparin. Subcutaneous dalteparin 5000 IU was administered once daily from day 1 until full mobilization. All patients received postoperative analgesic treatment by means of intravenous fentanyl, alfentanil, and oxycodone as well as oral oxycodone.

The cardiac output, measured as cardiac index was monitored in the first 12 hours after surgery. The Swan-Ganz catheter was removed before the transfer to the primary unit. Patients who required regular oxycodone treatment were prescribed macrogols and bisacodyl from day 1 to prevent constipation.

All patients gave written informed consent and the study was conducted in agreement with the Declaration of Helsinki. The Central Denmark Region Committees on Health Research (1-10-72-179-15) and the Danish Data Protection Agency (ID: 1765, case number: 1-16-02-403-15) approved the study before initiation.

\section{Statistics}

Continuous data are presented as median, 25 th and 75 th percentiles, and range when data were non-normally distributed or mean $\pm \mathrm{SD}$ and 


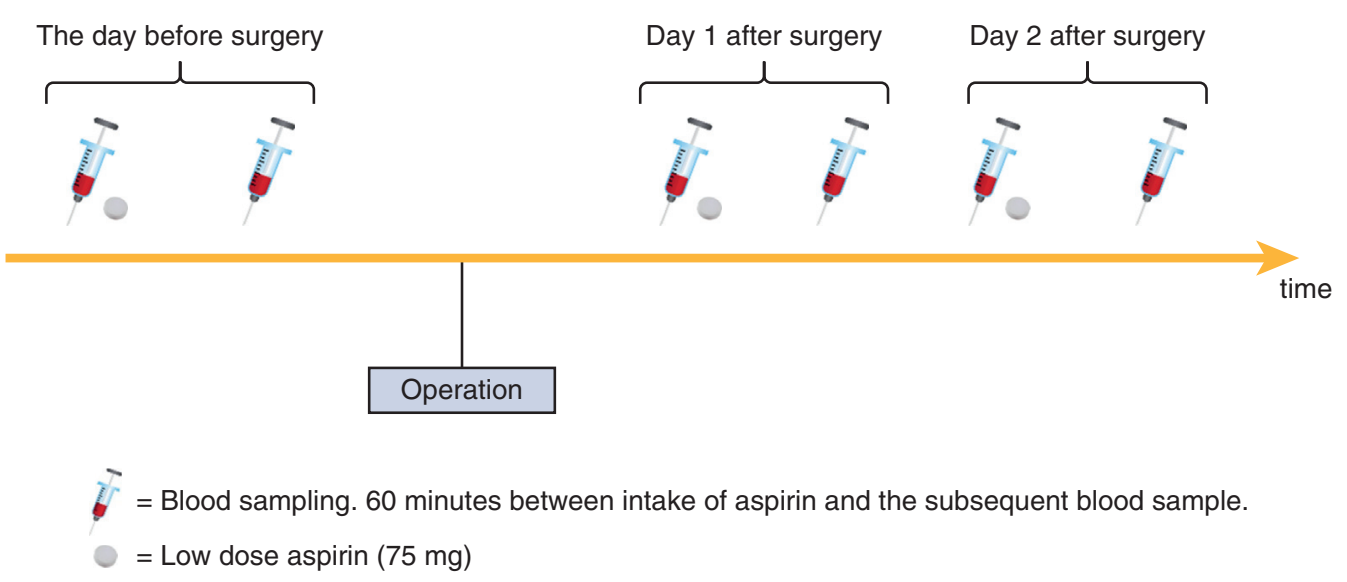

FIGURE 1. Blood sample flow chart.

confidence intervals if data were normally distributed. Differences in biochemical parameters between preoperative and postoperative data as well as data from before and after intake of aspirin were analyzed with paired $t$ test for normally distributed data and with Wilcoxon signed rank test when data were non-normally distributed. A 2-tailed $P$ value $<.05$ was considered statistically significant. An unpaired $t$ test analysis was used to test whether patients receiving proton pump inhibitors differed from patients not receiving proton pump inhibitors. Analyzing the correlation between levels of SA and Multiplate Analyzer (Roche, Roche Diagnostics, Mannheim, Germany) and VerifyNow Aspirin Test (Accumetrics, San Diego, Calif), was conducted using the Pearson correlation coefficient $(r)$. Changes in platelet aggregation on day 1 from before aspirin intake to $1,2,3$, and 4 hours after aspirin intake were analyzed using the Friedman test because the data did not fulfill the prerequisites for performing analysis of variance repeated measurements.

The primary outcome was the difference in change of SA concentration before and 1 hour after intake of aspirin in a comparison of preoperative data with data from day 1 (eg, comparing a mean to a known value). Using data from patients with cardiovascular disease published by Rubak and colleagues, ${ }^{2}$ we estimated the differences in mean concentration of SA change between preoperative and day 1 , before and 1 hour after intake to $4.00 \mu \mathrm{g} / \mathrm{mL}$ with an SD of $1.30 \mu \mathrm{g} / \mathrm{mL}$. We chose a minimum relevant difference of $0.70 \mu \mathrm{g} / \mathrm{mL}$, and with a significance level of $(2 \alpha)$ at .05 and a power $(1-\beta)$ at $90 \%$, a sample size of 37 patients was needed. To ensure a complete data set we chose to include 43 patients, and this was because the substudy expanded to 53 patients.

\section{Laboratory Investigations}

SA measurements. The main metabolite of aspirin, SA, was measured using liquid chromatography-electrospray-ionization-tandem mass spectrometry (LC-ESI-MS/MS) using pneumatically assisted electrospray ionization as previously described by Sorensen and colleagues. ${ }^{11}$ Blood samples were collected in chilled lithium heparin tubes and plasma was separated using centrifugation $(3000 \mathrm{~g}$ for 10 minutes at $4^{\circ} \mathrm{C}$ ) and stored at $-80^{\circ} \mathrm{C}$ until analyzed.

Serum $\mathbf{T x B}_{2}$. Serum $\mathrm{TxB}_{2}$ concentrations were measured using a commercially available enzyme-linked immunosorbent assay according to the manufacturer's instructions (Cayman Chemical, Ann Arbor, Mich), with the modification that serum was collected from whole blood using centrifugation ( $2600 \mathrm{~g}$ for 10 minutes) after 30 minutes until 1 hour of clotting at $37^{\circ} \mathrm{C}$. All samples were analyzed in duplicate, and samples yielding results outside the $20 \%$ to $80 \%$ range of the standard curve were reassayed with appropriate dilutions.

Platelet aggregation tests. Platelet aggregation was evaluated using multiple electrode aggregometry using Multiplate Analyzer aggregometer using arachidonic acid (ASPI) test $0.5 \mathrm{mM}$, collagen (COL) test $3.2 \mu \mathrm{g} / \mathrm{mL}$, adenosine diphosphate $6.5 \mu \mathrm{M}$ and thrombinreceptor-activated peptide- $6(32 \mu \mathrm{M})$ as agonists. Furthermore, platelet aggregation was analyzed using the VerifyNow Aspirin Test assay. Platelet aggregation was expressed as area under the curve (aggregation units $\times$ minutes) using a Multiplate Analyzer and as aspirin reaction units using VerifyNow Aspirin Test. Blood samples for the Multiplate Analyzer were collected in tubes containing hirudin and in tubes containing $3.2 \%$ sodium citrate for VerifyNow Aspirin Test. Reference intervals used for the Multiplate Analyzer were derived from the utilized laboratory ${ }^{12,13}$ and the range for the VerifyNow Aspirin Test, was supplied by the manufacturer (Accumetrics).

Other laboratory tests. Blood hemoglobin, platelet count, immature platelet fraction, and immature platelet count were determined using the XE-5000 hematology analyzer (Sysmex, Kobe, Japan). Plasma fibrinogen (functional) and fibrin D-dimer were measured using CS2100i (Sysmex). von Willebrand factor (antigen) and coagulation factor VIII (functional) were measured using the ACL TOP (ILS Laboratories, Bedford, Mass). Plasma creatinine and estimated glomerular filtration rate were measured using Cobas 6000 (Roche Diagnostics, Indianapolis, Ind). Plasma copeptin was determined using the Copeptin proAVP KRYPTOR assay (Brahms, Henningsdorf, Germany)

\section{RESULTS (VIDEO 1)}

We invited 149 patients who met the inclusion criteria (Figure 2). In total, 67 patients gave consent and were enrolled and 53 patients were included in the study without protocol deviations. SA was measured before and 1 hour after intake of aspirin preoperatively and at day 1 and day 2 with a median of 60 minutes (interquartile range: 5961 minutes).

Table 1 shows the characteristics of the study population. Most patients were male with a low predicted perioperative mortality as indicated by a European System for Cardiac Operative Risk Evaluation Score median (25th-75th percentiles) below $1 \%(0.8-1.2)$. In total, 13 patients were treated with proton pump inhibitors (eg, pantoprazole) before surgery as well as on day 1 and day 2 . Only 1 patient required a norepinephrine infusion pressure of $>0.1 \mu \mathrm{g} / \mathrm{kg} / \mathrm{min}$ in the immediate postoperative period, and no patients were transferred to the primary unit if they continued to require 


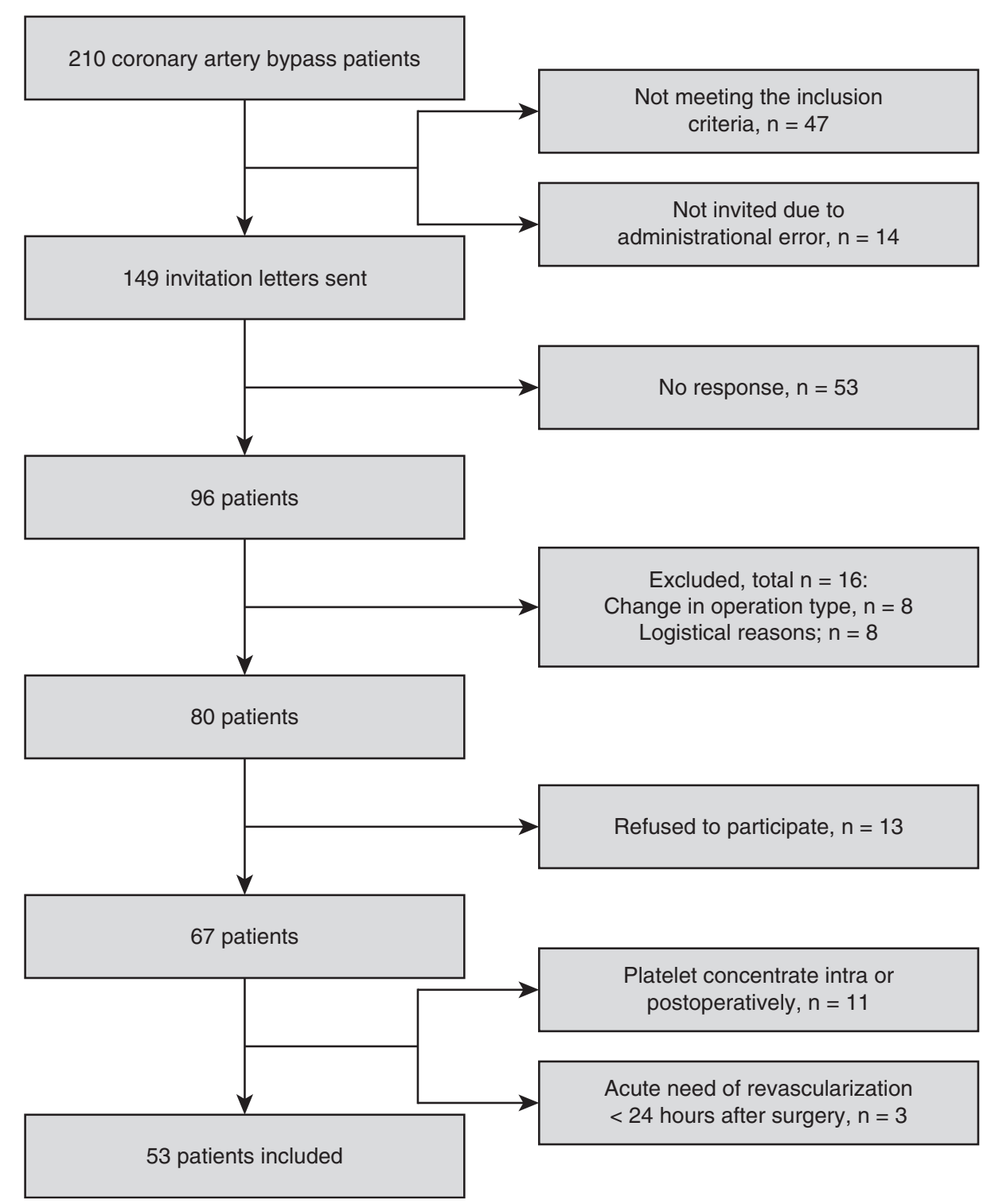

FIGURE 2. Consolidated Standards of Reporting Trials diagram illustrating the inclusion and exclusion process.

vasopressor infusion. Table 2 shows an overall normal biochemical profile before aspirin intake on the day before surgery. At postoperative day 1 and 2, von Willebrand factor, coagulation factor VIII, fibrinogen, and copeptin levels were elevated.

SA and $\mathbf{T x B}_{2}$. In a comparison of the differences in SA concentration before and 1 hour after intake of aspirin on the day before surgery with day 1 and day 2 after surgery, a significantly reduced increase in SA plasma level after the intake of aspirin was found at day 1 and 2 $(P<.0001$; Table 3$)$. Furthermore, the absolute SA levels 1 hour after intake of aspirin on day 1 and day 2 were significantly lower than the respective preoperative SA levels. The differences in SA levels were $4.18 \mu \mathrm{g} / \mathrm{mL}$ on the day before surgery, $1.63 \mu \mathrm{g} / \mathrm{mL}$ on day 1 , and $2.75 \mu \mathrm{g} / \mathrm{mL}$ on day 2 . In the subgroup, differences in SA levels on day 1 were $1.02 \mu \mathrm{g} / \mathrm{mL}$ (1 hour), $2.42 \mu \mathrm{g} / \mathrm{mL}$ ( 2 hours), $1.78 \mu \mathrm{g} / \mathrm{mL}$ ( 3 hours), and $1.14 \mu \mathrm{g} / \mathrm{mL}$ (4 hours). In a comparison of the change in concentration of SA from before aspirin intake to after aspirin intake on day 1 in the subgroup of patients at 2, 3, and 4 hours after intake, a significantly reduced increase in SA plasma levels after intake of aspirin were still present $(P$ values of $<.001$, $<.0001$, and $<.0001$, respectively; Table 3 ).

Patients treated with proton pump inhibitors (eg, pantoprazole) did not differ in SA levels compared with patients not treated with proton pump inhibitors. The mean differences (interquartile range) in SA levels in patients treated 
TABLE 1. Characteristics of the study population $(n=53)$

\begin{tabular}{lc}
\multicolumn{1}{c}{ Variable } & Value \\
\hline Age, y & $67(61-72)$ \\
Male sex & $46(87)$ \\
Body mass index & $27.7(25.9-30.4)$ \\
Smoker & $11(21)$ \\
Blood drainage $\leq 24$ h, mL & $700(500-913)$ \\
Cardiopulmonary bypass time, & $84(65-98)$ \\
$\quad$ minutes & \\
Euroscore II, \% & $0.94(0.8-1.2)$ \\
Comorbidity* & \\
$\quad$ Hypertension & \\
$\quad$ Hypercholesterolemia & \\
Diabetes & $51(93)$ \\
\hline
\end{tabular}

Data are presented as median (25th-75th percentile) or $\mathrm{n}(\%)$. On the basis of prescribed medicine.

with proton pump inhibitors before surgery were $4.18 \mu \mathrm{g} / \mathrm{mL}$ (3.86-5.60) on the day before surgery, $1.83 \mu \mathrm{g} / \mathrm{mL}(1.15-$ 2.48) on day 1 , and $2.59 \mu \mathrm{g} / \mathrm{mL}(1.51-3.24)$ on day 2 .

Compliance with aspirin intake before surgery was confirmed by low serum $\mathrm{TxB}_{2}$ levels (Table 3 ). The levels were below $30 \mathrm{ng} / \mathrm{mL}$, corresponding to more than $95 \%$ inhibition of platelet COX-1 activity.

Preoperatively, $\mathrm{TxB}_{2}$ concentration levels decreased significantly $(P<.0001)$ after intake of aspirin. The $\mathrm{TxB}_{2}$ levels were very low on day 1 (Table 3 ), and no difference between the levels in $\mathrm{TxB}_{2}$ concentration were found after intake of aspirin $(P=.70)$. On day $2, \mathrm{TxB}_{2}$ concentration levels again decreased significantly $(P<.0001)$ after intake of aspirin. Levels of $\mathrm{TxB}_{2} 1$ hour after intake of aspirin were $1.62 \mathrm{ng} / \mathrm{mL}$ preoperatively, $0.52 \mathrm{ng} / \mathrm{mL}$ on day 1 , and $2.54 \mathrm{ng} / \mathrm{mL}$ on day 2 .

All patients, except for 5, received the nonsteroidal antiinflammatory COX-1 selective inhibitor drug ketorolac
(Toradol; Roche, Basel, Switzerland) with the ability to inhibit platelet COX, before the blood sample on day 1 . The 5 patients who did not receive ketorolac had a slight, nonsignificant, decrease in $\mathrm{TxB}_{2}$ concentration after intake of aspirin on day $1(P=.13)$.

\section{Platelet Aggregation}

The changes in platelet aggregation are depicted in Figure 3. Preoperatively, a significant decrease in platelet aggregation was found after intake of aspirin determined according to both methods. Postoperatively at day 1, neither the Multiplate Analyzer ASPI test, nor VerifyNow Aspirin Test showed a significant decrease in platelet aggregation after aspirin intake. Similar results were found in the subgroup of patients who had additional blood samples obtained 2, 3, and 4 hours after aspirin intake ( $P$ values $>.14)$.

On day 2, the platelet aggregation showed the same pattern as preoperatively with a significant decrease in platelet aggregation after intake of aspirin $(P<.001)$. A significantly higher platelet aggregation was detected in a comparison of the preoperative levels before intake of aspirin with the postoperative levels before intake of aspirin on day 2, determined using the Multiplate Analyzer ASPI test $(P<.0001)$, and VerifyNow Aspirin Test $(P<.0001)$. Regarding the nonaspirin-specific tests, platelet aggregation measured using the COL test increased significantly in the postoperative period $(P<.0001)$. Platelet aggregation measured using thrombin receptor-activated peptide- 6 and adenosine diphosphate decreased significantly in the postoperative period $(P<.0001)$. All aspirin nonspecific tests changed within the reference interval as depicted in Table 4. Using the Multiplate Analyzer ASPI test, the platelet aggregation was below the reference interval throughout the study period because patients were treated with aspirin. A significant, though weak, correlation was found between levels of SA and the Multiplate Analyzer ASPI test

TABLE 2. Biochemical characteristics before aspirin preoperatively, and on day 1 and day 2 postoperatively $(\mathbf{n}=53)$

\begin{tabular}{|c|c|c|c|c|}
\hline Variable & Reference interval & Preoperative & Day 1 & Day 2 \\
\hline Hemoglobin, $\mathrm{mmol} / \mathrm{L}$ & $7.3-10.5^{*}$ & $8.8(8.4-9.2)$ & $6.7(6.2-7.3)$ & $6.5(5.9-6.9)$ \\
\hline Hematocrit & $0.35-0.50^{*}$ & $0.42(0.40-0.44)$ & $0.33(0.30-0.35)$ & $0.31(0.28-0.34)$ \\
\hline Creatinine, $\mu \mathrm{mol} / \mathrm{L}$ & $45-105^{*}$ & $81(71-95)$ & $82(67-89)$ & $85(71-97)$ \\
\hline Platelet count, $10^{9} / \mathrm{L}$ & $145-390^{*}$ & $225(197-258)$ & $167(137-200)$ & $149(129-179)$ \\
\hline Immature platelet fraction & $0.011-0.061$ & $0.032(0.024-0.043)$ & $0.041(0.029-0.054) \dagger$ & $0.042(0.029-0.051) \dagger$ \\
\hline Immature platelet count, $10^{9} / \mathrm{L}$ & $2.5-16.6$ & $7.5(6.0-10.2)$ & $6.9(4.9-8.9) \dagger$ & $6.0(4.18-8.25) \dagger$ \\
\hline von Willebrand factor (antigen), $10^{3} \mathrm{IU} / \mathrm{L}$ & $0.35-1.71$ & $1.67(1.20-2.10)$ & $2.21(2.12-2.84)$ & $2.84(2.25-3.35)$ \\
\hline Factor VIII (functional), $10^{3} \mathrm{IU} / \mathrm{L}$ & $0.66-1.55$ & $1.29(1.15-1.49)$ & $1.68(1.37-1.98)$ & $1.97(1.70-2.29)$ \\
\hline Fibrinogen, $\mu \mathrm{mol} / \mathrm{L}$ & $5.5-12.0$ & $10.3(9.0-11.4)$ & $10.3(9.4-12.1)$ & $15.4(14.1-17.5)$ \\
\hline Fibrin D-dimer (FEU), mg/L & $<0.50$ & $0.47(0.29-0.68)$ & $0.71(0.48-1.00)$ & $0.52(0.45-0.84)$ \\
\hline Copeptin $(\mathrm{n}=43), \mathrm{pmol} / \mathrm{L}$ & $5.0(2.0) \ddagger$ & $5.7(3.7-9.4)$ & $46.6(26.6-72.8)$ & $12.8(7.6-23)$ \\
\hline
\end{tabular}

FEU, Fibrinogen equivalent units. *Reference interval merged for both sexes. Data are shown as median (25th-75th percentile) and ranges because of non-Gaussian distribution for some of the parameters. $†$ Missing data for 1 patient. $\ddagger$ Mean \pm (SD). Age matched (65-80 years) according to baseline characteristics of the study population. ${ }^{29}$ 


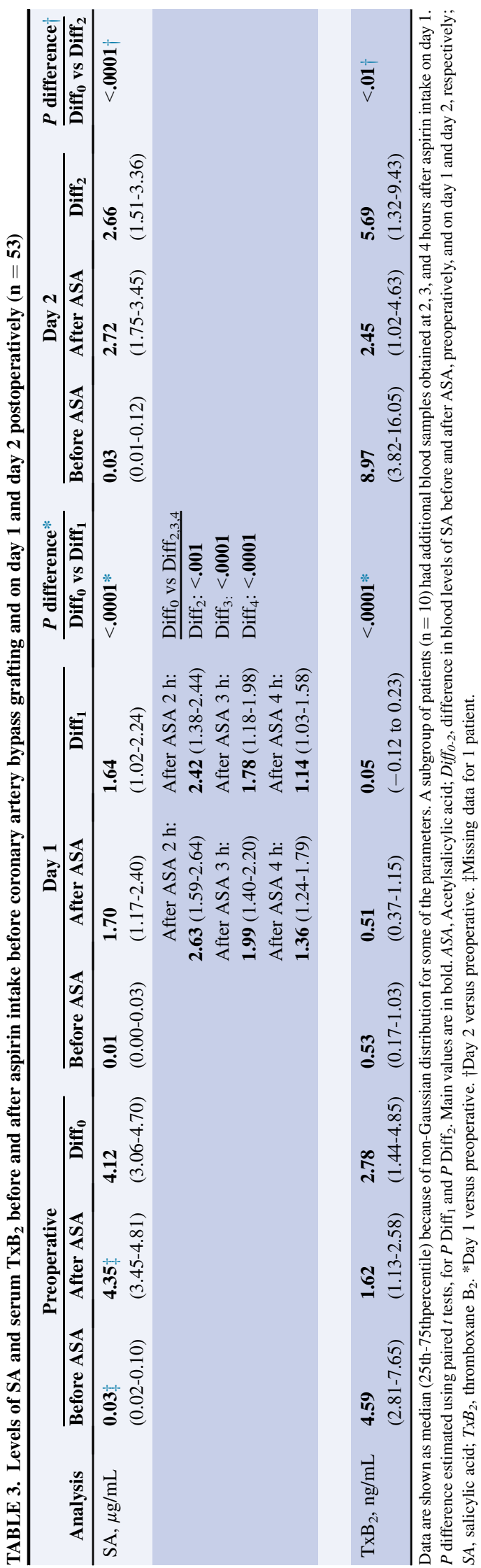

$(P<.0001 ; r=-0.26)$ and the VerifyNow Aspirin Test

$(P=.0001 ; r=-0.24)$.

\section{Other Laboratory Tests}

von Willebrand factor, coagulation factor VIII, and fibrinogen all showed a significant increase in the postoperative period compared with preoperative levels $(P<.0001)$, especially on day 2 . Levels of copeptin increased substantially on day 1 compared with the day before surgery, but almost returned to normal on day 2 (Table 2).

\section{DISCUSSION}

This study showed that the absorption of aspirin is reduced in the early postoperative period of CABG. The lack of increase of SA and reduced effect with regard to platelet inhibition after aspirin intake was particularly pronounced on day 1 after CABG.

Aspirin is a quick-acting and quickly eliminated drug. ${ }^{14}$ Levels of aspirin and SA in patients with cardiovascular disease measured over 24 hours showed that aspirin and SA are almost nondetectable as early as 6 hours after administration of the drug. ${ }^{2}$

We measured SA using LC-ESI-MS/MS because this is a validated method known for its superiority and detection efficacy at low therapeutic concentrations for drugs such as aspirin. ${ }^{11}$ Using LC-ESI-MS/MS measurements of SA, the free fraction of SA and the protein-bound fraction of SA is measured. This eliminates the possibility that an increased protein binding of SA after surgery could explain the significantly lower SA concentrations found the first and second day after surgery. Consequently, the reduced level of SA after CABG measured in the present study most likely reflects a reduced ability to absorb aspirin from the gastrointestinal tract.

Although the pharmacokinetics of aspirin are well described in humans under normal conditions, ${ }^{15}$ little is known about the effect of surgery on the absorption of the drug. A study conducted by Zhang and colleagues showed that the absorption of aspirin might be impaired early after acute myocardial infarction. ${ }^{16}$ Despite strong evidence that the antiplatelet effect of aspirin is compromised in the early postoperative period of $\mathrm{CABG},{ }^{17}$ to our knowledge, no previous study has investigated aspirin absorption. However, findings showing reduced bioavailability of metoprolol after $\mathrm{CABG}$ and measured using liquid chromatographytandem mass spectrometry, ${ }^{18}$ supports the hypothesis of alteration of gastrointestinal absorption after CABG. Patients in the present study did not have food intake between blood samples before and 1 hour after intake of aspirin on day 1 and 2 after CABG and all patients received opioid treatment. Thus, our finding might be partly explained by impaired gastric emptying rate, reduced food intake, or impaired peristalsis because of opioid treatment. 


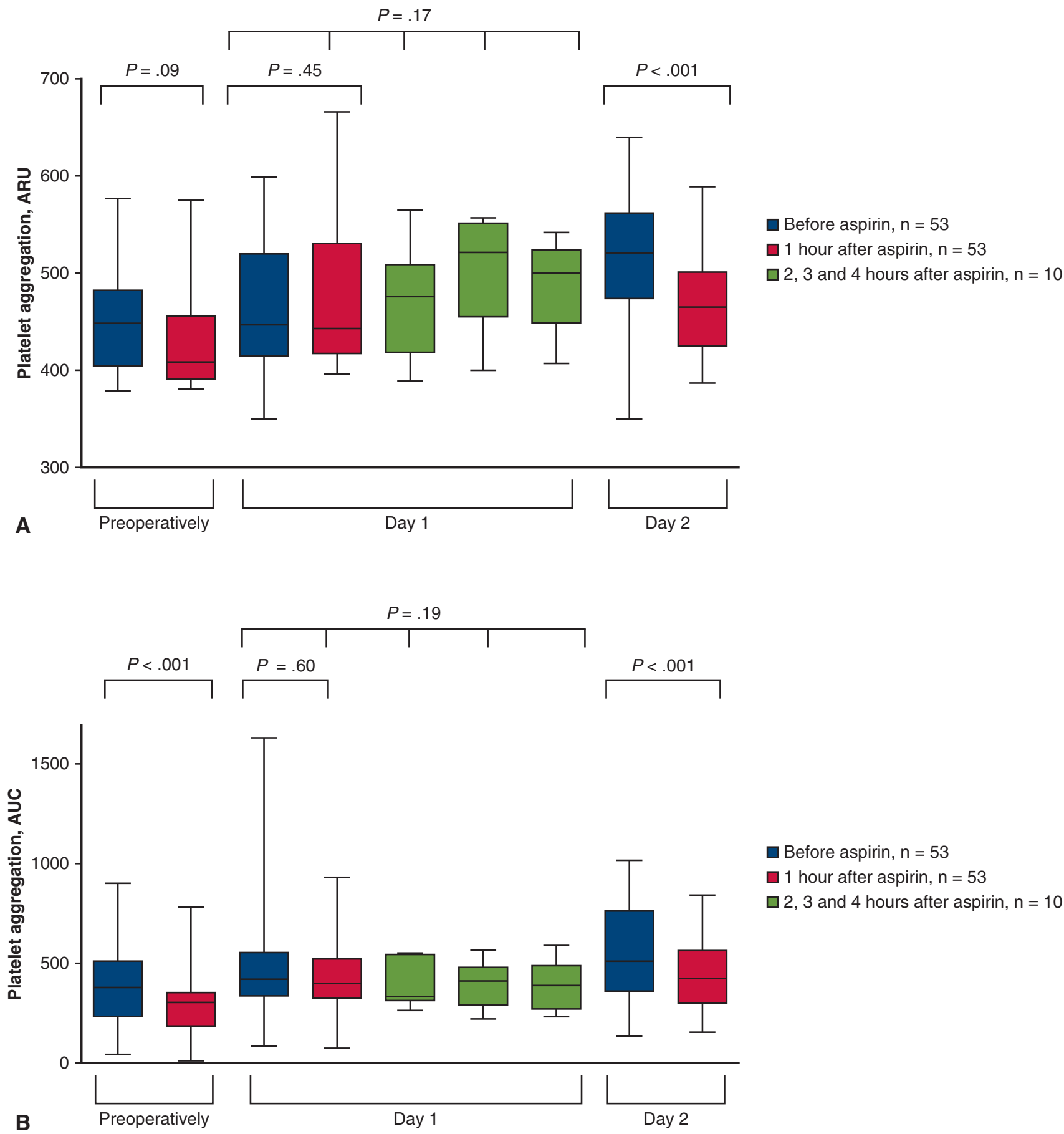

FIGURE 3. A, Platelet aggregation determined using a Multiplate Analyzer (Roche, Roche Diagnostics, Mannheim, Germany) with the agonist arachidonic acid (ASPI) test as area under the curve $(A U C)$. Boxes indicate the median and 25 th and 75 th percentiles, and whiskers indicate range $(\mathrm{n}=53)$. B, Platelet aggregation determined using the VerifyNow Aspirin Test (Accumetrics, San Diego, Calif) as aspirin reaction units (ARU). Boxes indicate the median and 25th and 75th percentiles, and whiskers indicate range $(n=53)$. Because of technical reasons, 2 patients were excluded at the time of blood samples taken preoperatively before intake of aspirin and 1 hour after intake of aspirin, respectively. Two patients were excluded at the time of blood samples taken at day 1 before intake of aspirin and 1 patient was excluded at the time of blood samples taken at day 1, 1 hour after intake of aspirin.

Numerous clinical trials have investigated whether some patients are aspirin-resistant or nonresponders, and has been substantially debated in recent years. ${ }^{19}$ Reduced response to aspirin is today believed primarily to be related to noncompliance. ${ }^{20}$ However, in the present study aspirin compliance was confirmed by low $\mathrm{TxB}_{2}$ level and observed intake of aspirin. 
TABLE 4. Platelet aggregation values for Multiplate Analyzer (AUC) and VerifyNow Aspirin Test (ARU)

\begin{tabular}{|c|c|c|c|c|c|c|c|c|c|c|}
\hline \multirow[b]{2}{*}{ Aggregation parameter } & \multirow[b]{2}{*}{ Ref.-interval } & \multicolumn{3}{|c|}{ Preoperative } & \multicolumn{3}{|c|}{ Day 1} & \multicolumn{3}{|c|}{ Day 2} \\
\hline & & Before ASA & After ASA & $P$ value & Before ASA & After ASA & $P$ value & Before ASA & After ASA & $P$ value \\
\hline \multicolumn{11}{|l|}{ Multiplate Analyzer } \\
\hline ASPI & $685-1323$ & $384(190)$ & 298 (174) & $<.001$ & $455(231)$ & $422(167)$ & .29 & $542(231)$ & 447 (182) & $<.001$ \\
\hline $\mathrm{COL}$ & $560-1070$ & $711(192)$ & $744(218)$ & .11 & $866(264)$ & $875(251)$ & .78 & $784(283)$ & $765(240)$ & .53 \\
\hline $\mathrm{ADP}$ & $483-1173$ & $982(265)$ & $964(262)$ & .53 & $958(286)$ & 992 (277) & .15 & 825 (228) & 855 (219) & .25 \\
\hline TRAP & $842-1630$ & $1468(223)$ & $1481(271)$ & .63 & 1459 (307) & $1424(312)$ & .34 & 1157 (284) & $1150(240)$ & .83 \\
\hline \multirow[t]{2}{*}{ VerifyNow } & Measurement rang & & & & & & & & & \\
\hline & $350-700$ & $452(55)$ & $432(53)$ & .09 & $469(63)$ & $475(70)$ & .45 & $510(66)$ & $467(50)$ & $<.001$ \\
\hline
\end{tabular}

Data are shown as mean (SD). $P$ values calculated as before versus after ASA for each of the 3 days $(\mathrm{n}=53)$. Paired $t$ tests were used for all of the assays except for the Multiplate Analyzer (Roche, Roche Diagnostics, Mannheim, Germany) ASPI test at day 1, where a Wilcoxon test was used because of non-Gaussian distribution. VerifyNow Aspirin Test is from Accumetrics, San Diego, California. Ref.-interval, Reference interval; ASA, acetylsalicylic acid; ASPI, arachidonic acid; COL, collagen; ADP, adenosine diphosphate; TRAP, thrombin activating peptide-6.

It has been known for decades that SA is more rapidly absorbed at low $\mathrm{pH}$ values. ${ }^{21}$ However, the gastric $\mathrm{pH}$ after $\mathrm{CABG}$ in the early postoperative period is normalized 24 hours after surgery. ${ }^{22}$ Therefore, it is unlikely that surgery-induced $\mathrm{pH}$ alterations in the gastrointestinal tract could explain the reduced aspirin absorption found in the present study.

The observed low levels of $\mathrm{TxB}_{2}$ on day 1 are most likely explained by the administration of ketorolac (Toradol). Ketorolac is a nonsteroidal anti-inflammatory drug that reduces postoperative pain ${ }^{23}$ and is known to reduce $\mathrm{TxB}_{2}$ levels. Ketorolac was only administered once, or in a few patients twice, before extubation on day 0 , hence affecting $\mathrm{TxB}_{2}$ levels on day 1 . Despite the fact that most patients received ketorolac known to inhibit platelet aggregation, no reduction in platelet aggregation was found after aspirin intake on day 1 after surgery and could not have affected the observed differences in platelet aggregation on day 1 . Further, ketorolac did not affect the plasma levels of SA.

Preoperatively, a significant decrease in platelet aggregation after intake of aspirin was shown according to the

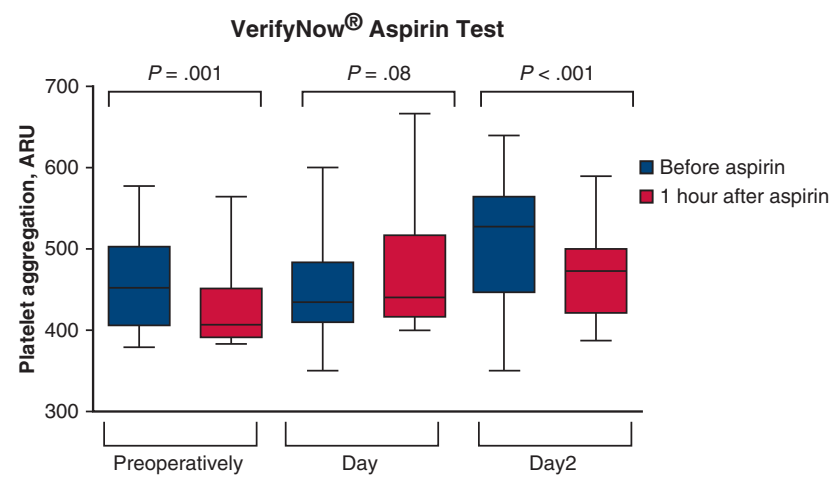

VIDEO 1. The absorption of aspirin is reduced after coronary artery bypass grafting: main results outlined by the authors. Video available at: https://www.jtcvs.org/article/S0022-5223(18)32482-6/fulltext.
Multiplate Analyzer ASPI test and VerifyNow Aspirin Test. Remarkably, no significant reduction in platelet aggregation was found after aspirin intake on day 1 after surgery. This was also the case 4 hours after aspirin intake indicating that it is merely a question of reduced effect than just delayed effect. In the postoperative period, a significant increase in the levels of von Willebrand factor, factor VIII, and fibrinogen was shown. These findings indicate an acute phase response and resemble previous findings after cardiac and major surgery. ${ }^{24}$ On day 2 subsequent to surgery, the platelet inhibition was partly restored. The present study supports the recent finding by our group of a reduced effect of aspirin shortly after coronary revascularization without $\mathrm{CPB},{ }^{8}$ and the phenomenon of a reduced antiplatelet effect of aspirin early after CABG with CPB is well described. ${ }^{25}$ Earlier studies showed different results using different platelet aggregation assays when studying patients with stable coronary artery disease. ${ }^{4}$ Notably, in the present study both platelet aggregation assays showed the same pattern, which strongly supports our hypothesis.

A possible explanation for the reduced aspirin effect after surgery is the fact that patients received surgery with CPB. Recently it was shown that CPB reduces platelet aggregation in the immediate postoperative period after cardiac surgery. ${ }^{26}$ However, this finding is not supported by the present study.

The present study population had a postoperative significantly increased immature platelet fraction. This indicates that patients in the present study have a hyperactive circulating platelet population, because immature platelets are supposed to be more hemostatic-reactive and are largely unaffected by aspirin because of their COX-2 expression. $^{27}$

Some limitations have to be addressed. First, we recognize that we used biochemical end points and our study was not sufficiently powered to investigate whether reduced absorption of aspirin was associated with an increased risk of thromboembolic events. Second, the design of our study 
did not allow any conclusion regarding etiological explanations for the reduced absorption of aspirin. Third, most patients included in the study were men $(87 \%)$, thereby not completely reflecting the usual sex ratio for patients who undergo CABG. ${ }^{10}$ However, there is no reason to believe that absorption differs between the 2 sexes.

We cannot exclude that propofol and tranexamic acid influence platelet aggregation. ${ }^{28}$ However, to our knowledge, no literature indicates that these medications affect aspirin absorption, which is the main focus in the present study. Urinary alkalization increases the clearance of salicylate. We did not investigate the urinary $\mathrm{pH}$, however, we expect the urinary $\mathrm{pH}$ to be within physiological limits in the setting of elective CABG surgery with tightly monitored acid-base equilibrium. Moreover, the patients were not treated with drugs on day 1 and day 2 that could affect the urine $\mathrm{pH}$, such as infusions of $\mathrm{NaCl}$ or $\mathrm{NaHCO}_{3}$.

\section{CONCLUSIONS}

The present study shows for the first time that the absorption of aspirin as measured by the inactive metabolite SA is impaired in the early postoperative course after CABG. Impaired absorption might represent the main cause of the well known reduced antiplatelet effect of aspirin in the early postoperative phase. This finding challenges the current practice to resume aspirin prophylaxis after CABG with low-dose oral administration. We suggest that future studies investigate the resumption of aspirin treatment administered intravenously to bypass the impaired gastrointestinal absorption. However, large-scale clinical trials are warranted to investigate the effect of intravenous aspirin treatment and the correlation with thromboembolic events in the early postoperative period of CABG.

\section{Conflict of Interest Statement}

Anne-Mette Hvas has received speaker's fees from CSL Behring, Bayer, Boehringer-Ingelheim, Bristol-Myers Squibb, and Leo Pharma, has received unrestricted research support from Octapharma, CSL Behring, and Leo Pharma, and has participated in advisory board for Novo Nordisk. All other authors have nothing to disclose with regard to commercial support.

The authors acknowledge laboratory technicians Vivi Bo Mogensen and Mai Stenulm Veirup for their assistance in the laboratory. We thank Tore Hardlei and Jørgen Bo Hasselstrøm, Department of Forensic Medicine, Aarhus University, Denmark, for analysis of SA and their perpetual professionalism. Furthermore, we send our gratitude to secretary Annette Sejr Lauridsen from Department of Cardiothoracic Surgery as well as all of the nurses at Department of Cardiothoracic Surgery and Department of Anaesthesiology and Intensive Care for making the project possible. Finally, assistance in producing our video provided by Torben Hoffman is greatly appreciated.

\section{References}

1. Windecker S, Kolh P, Alfonso F, Collet JP, Cremer J, Falk V, et al. 2014 ESC/ EACTS guidelines on myocardial revascularization: The task force on myocardial revascularization of the European Society of Cardiology (ESC) and the European Association for Cardio-Thoracic Surgery (EACTS) developed with the special contribution of the European Association of Percutaneous Cardiovascular Interventions (EAPCI). Eur Heart J. 2014;35:2541-619.

2. Rubak P, Hardlei TF, Wurtz M, Kristensen SD, Hvas AM. Low-dose acetylsalicylic acid therapy monitored with ultra high performance liquid chromatography. Clin Biochem. 2013;46:988-92.

3. Angiolillo DJ. The evolution of antiplatelet therapy in the treatment of acute coronary syndromes: from aspirin to the present day. Drugs. 2012;72:2087-116.

4. Grove EL, Hvas AM, Johnsen HL, Hedegaard SS, Pedersen SB, Mortensen J, et al. A comparison of platelet function tests and thromboxane metabolites to evaluate aspirin response in healthy individuals and patients with coronary artery disease. Thromb Haemost. 2010;103:1245-53.

5. Nalysnyk L, Fahrbach K, Reynolds MW, Zhao SZ, Ross S. Adverse events in coronary artery bypass graft $(\mathrm{CABG})$ trials: a systematic review and analysis. Heart. 2003;89:767-72

6. Tarakji KG, Sabik JF III, Bhudia SK, Batizy LH, Blackstone EH. Temporal onset, risk factors, and outcomes associated with stroke after coronary artery bypass grafting. JAMA. 2011;305:381-90.

7. Likosky DS, Marrin CA, Caplan LR, Baribeau YR, Morton JR, Weintraub RM, et al. Determination of etiologic mechanisms of strokes secondary to coronary artery bypass graft surgery. Stroke. 2003;34:2830-4.

8. Modrau IS, Wurtz M, Kristensen SD, Hvas AM. Reduced effect of aspirin and clopidogrel following hybrid coronary revascularization. Clin Appl Thromb Hemost. 2015;21:603-11.

9. Krasopoulos G, Brister SJ, Beattie WS, Buchanan MR. Aspirin "resistance" and risk of cardiovascular morbidity: systematic review and meta-analysis. BMJ. 2008:336:195-8.

10. Nashef SA, Roques F, Sharples LD, Nilsson J, Smith C, Goldstone AR, et al. EuroSCORE II. Eur J Cardiothorac Surg. 2012;41:734-44; discussion: 744-35.

11. Sorensen LK. Determination of acidic and neutral therapeutic drugs in human blood by liquid chromatography-electrospray tandem mass spectrometry. Forensic Sci Int. 2011;206:119-26.

12. Rubak P, Villadsen K, Hvas AM. Reference intervals for platelet aggregation assessed by multiple electrode platelet aggregometry. Thromb Res. 2012;130:420-3.

13. Pedersen OH, Larsen ML, Grove EL, van Kooten Niekerk PB, Bønløkke S, Nissen $\mathrm{PH}$, et al. Platelet characteristics in patients with essential thrombocytosis. Cytometry B Clin Cytom. May 23, 2018 [Epub ahead of print].

14. Bell DS. Aspirin in the prevention of cardiovascular events in patients with diabetes. Postgrad Med. 2016;128:180-90.

15. Graham G, Roberts MS, Day RO, Rainsford KD. Aspirin and Related Drugs. Boca Raton, FL: CRC Press; 2004.

16. Zhang CL, Wilson KM, Stafford I, Bochner F, Horowitz JD. Absorption kinetics of low dose aspirin in patients with evolving acute myocardial infarction. Drug Invest. 1994; 7:169-74.

17. Zimmermann N, Kurt M, Winter J, Gams E, Wenzel F, Weber AA, et al. Aspirin induced platelet inhibition in patients undergoing cardiac surgery. Platelets. 2007;18:528-34.

18. Valtola A, Kokki H, Gergov M, Ojanpera I, Ranta VP, Hakala T. Does coronary artery bypass surgery affect metoprolol bioavailability. Eur J Cin Pharmacol. 2007;63:471-8.

19. Klasic A, Lakusic N, Gaspar L, Kruzliak P. The monitoring of antiaggregation effect of acetylsalicylic acid therapy by measuring serum thromboxane B2 in patients with coronary artery bypass grafting. Blood Coagul Fibrinolysis. 2016;27: 370-3.

20. Bagoly Z, Homorodi N, Kovacs EG, Sarkady F, Csiba L, Edes I, et al. How to test the effect of aspirin and clopidogrel in patients on dual antiplatelet therapy? Platelets. 2016;27:59-65.

21. Truitt EB Jr, Morgan AM. Gastrointestinal factors in aspirin absorption. A quantitative study. J Pharm Sci. 1963;53:129-34.

22. Ohki S, Kunimoto F, Isa Y, Tsukagoshi H, Ishikawa S, Ohtaki A, et al. Changes in gastric intramucosal $\mathrm{pH}$ and circulating blood volume following coronary artery bypass grafting. Can J Anaesth. 2000;47:516-21.

23. Bainbridge D, Cheng DC, Martin JE, Novick R. NSAID-analgesia, pain control and morbidity in cardiothoracic surgery. Can J Anaesth. 2006;53: 46-59.

24. Sartori TM, Maurizio PG, Sara P, Ugolino L, Annalisa A, Panagiotis T, et al Relation between long-term steroid treatment after heart transplantation, 
hypofibrinolysis and myocardial microthrombi generation. J Heart Lung Transplant. 1999; 18:693-700.

25. Zimmermann N, Kurt M, Wenk A, Winter J, Gams E, Hohlfeld T. Is cardiopulmonary bypass a reason for aspirin resistance after coronary artery bypass grafting? Eur J Cardiothorac Surg. 2005;27:606-10.

26. Paparella D, Brister SJ, Buchanan MR. Coagulation disorders of cardiopulmonary bypass: a review. Intensive Care Med. 2004;30:1873-81.

27. Guthikonda S, Lev EI, Patel R, DeLao T, Bergeron AL, Dong JF, et al. Reticulated platelets and uninhibited COX-1 and COX-2 decrease the antiplatelet effects of aspirin. J Thromb Haemost. 2007;5:490-6.
28. Fourcade O, Simon MF, Litt L, Samii K, Chap H. Propofol inhibits human platelet aggregation induced by proinflammatory lipid mediators. Anesth Analg. 2004;99:393-8. table of contents.

29. Morgenthaler NG, Struck J, Alonso C, Bergmann A. Assay for the measurement of copeptin, a stable peptide derived from the precursor of vasopressin. Clin Chem. 2006;52:112-9.

Key Words: CABG, aspirin, absorption, coagulation, platelets 